\title{
Diferenciales intra-urbanos del suicidio: el rol de la fragmentación social en Argentina
}

\author{
Intra-urban differentials of suicide: \\ the role of social fragmentation in Argentina
}

Carlos Marcelo Leveau (https://orcid.org/0000-0001-6240-9811) ${ }^{1}$

Carlos Guevel (https://orcid.org/0000-0001-8985-1255) ${ }^{1}$

Marcio Alazraqui (https://orcid.org/0000-0002-6507-0208) ${ }^{1}$

${ }^{1}$ Instituto de Producción, Economía y Trabajo, Consejo Nacional de Investigaciones Científicas y Técnicas, Universidad Nacional de Lanús. 29 de Septiembre 3901, Remedios de Escalada. B1824PJU Provincia de Buenos Aires Argentina. cmleveau@hotmail.com

\begin{abstract}
Suicide is one of the leading causes of death from injury in Argentina, and there are few studies analyzing intra-urban geographic variations in developing countries. The scope of this study was to analyze the relationship between suicide and socioeconomic status and social fragmentation in small areas of the Autonomous City of Buenos Aires (CABA) during the 2011-2015 period. An ecological study was conducted using data on suicide records in the resident population of the CABA available at the National Ministry of Security. A multiple spatial regression was carried out to test the relationship between suicide and indicators of socioeconomic status and social fragmentation by area. The risk of suicide was positively associated with higher indices of social fragmentation, whereas it was not related to the indices of poverty. The results of this study support the hypothesis of social fragmentation as a factor positively associated with the increase of intra-urban suicide in cities of developing countries.
\end{abstract}

Key words Suicide, Spatial analysis, Spatial regression, Urban areas
Resumen El suicidio constituye una de las principales causas de muerte por lesiones en Argentina y existen pocos estudios que analicen las variaciones geográficas intra-urbanas en países en desarrollo. El objetivo de este estudio fue analizar la relación entre el suicidio y el nivel socioeconómico y la fragmentación social en áreas pequeñas de la Ciudad Autónoma de Buenos Aires (CABA) durante el período 2011-2015. Se realizó un estudio ecológico utilizando datos sobre registros de suicidio en población residente de la CABA disponibles en el Ministerio de Seguridad de la Nación. Se llevó a cabo una regresión múltiple espacial para poner a prueba la relación entre el suicidio e indicadores de nivel socioeconómico y de fragmentación social por área. El riesgo de suicidio estuvo asociado positivamente a mayores indices de fragmentación social, mientras no mostró relación con el índice de pobreza. Los resultados de este estudio apoyan la hipótesis de fragmentación social como un factor asociado positivamente al aumento del suicidio a nivel intra-urbano en ciudades de países en desarrollo.

Palabras clave Suicidio, Análisis espacial, Regresión espacial, Áreas urbanas 


\section{Introducción}

En el mundo cada año se suicidan 800.000 personas y constituye la segunda causa de muerte en la población de 10 a 29 años ${ }^{1}$. En Argentina se registra un impacto importante de los suicidios en población joven. Dentro de las muertes por lesiones, el suicidio representa la segunda causa de muerte en 15 a 24 años y la tercera en importancia en población de 25 a 54 años $^{2}$. De acuerdo a este panorama, el suicidio representa un problema grave de salud pública, no solo a nivel nacional, sino también a escala mundial. La gravedad y complejidad de esta problemática requiere de enfoques de investigación multidisciplinarios que analicen los factores contextuales asociados a la ocurrencia de los suicidios ${ }^{1}$.

Desde el estudio pionero de Durkheim se ha postulado que tanto el nivel de integración social como el nivel de regulación social en un grupo social determinado tienen influencia sobre la frecuencia del suicidio ${ }^{3}$. Para Durkheim ${ }^{3}$ una sociedad se encuentra más integrada cuando las creencias y normas que la rigen tienen mayor influencia sobre los individuos que la componen. Instituciones sociales como el matrimonio, la familia, la iglesia o los grupos políticos promoverían una mayor integración y regulación social y actuarían como inhibidores del suicidio. De acuerdo a Durkheim, tanto las situaciones de exceso de integración social como de regulación social fueron predominantes en sociedades 'primitivas', por lo que estudios recientes han operacionalizado tanto los niveles de integración como de regulación social a través de un índice de fragmentación social ${ }^{4-6}$, construido a partir de variables que intentan captar procesos que generarían menor integración y regulación social, como una mayor movilidad poblacional y una menor importancia relativa del matrimonio y la familia en la conformación de los hogares de un área determinada. Es así como varios estudios empíricos parecen apoyar su proposición: el suicidio varía inversamente con el nivel de integración y regulación de los grupos sociales de los cuales el individuo forma una parte ${ }^{4-6}$.

Aunque el suicidio también es un fenómeno asociado a factores bioquímicos y psiquiátricos ${ }^{7}$, la relación entre el nivel de fragmentación social (lo opuesto a integración y regulación social) y el suicidio mostraría la importancia de lo social como su determinación, teniendo en cuenta el carácter complejo de este fenómeno. Desde la perspectiva de la determinación social en salud se considera que los niveles biológico y psicológico se encuentran subsumidos en el nivel social ${ }^{8}$. Si además se considera que lo social es simultáneamente espacial ${ }^{9}$, las desigualdades sociales en la salud se reflejarían en desigualdades en el territorio. Latinoamérica es la región más desigual del mundo ${ }^{10}$, por lo que al interior de sus mega-ciudades puede presentar zonas con niveles de desarrollo similares a ciudades de países desarrollados, pero también zonas con niveles mayores de pobreza no presentes en estos países. La Ciudad Autónoma de Buenos Aires (CABA) constituye un ejemplo de la convivencia de ambos extremos de desarrollo económico y social en una misma ciudad latinoamericana ${ }^{11}$. Por lo tanto, es esperable encontrar disparidades geográficas en el riesgo de suicidio a nivel intra-urbano en la CABA.

Si bien el riesgo de suicidio es mayor en áreas rurales con respecto a áreas urbanas ${ }^{12}$, hay algunos estudios geográficos que también mostraron un riesgo mayor en las áreas centrales (zonas de alta concentración de comercios y oficinas, también llamado distrito financiero) al interior de ciudades grandes ${ }^{13-17}$. Por lo tanto, el aumento de la urbanización puede generar nuevos contextos que propicien un aumento de los suicidios en las grandes ciudades. A nivel mundial, el aumento de los niveles de urbanización no ha sido homogéneo: el grupo de países menos desarrollados ha ido aumentando su ritmo de urbanización con respecto al grupo de países más desarrollados. Esta brecha de crecimiento fue dos veces mayor durante las décadas 1950 a 1970 y se proyecta que será cinco veces mayor en las próximas décadas ${ }^{18}$. Debido al crecimiento más acelerado de los niveles de urbanización fuera del mundo desarrollado, el alto nivel de población urbana (90\% vive en áreas urbanas de acuerdo al censo nacional de población de 2010) y el impacto del suicidio en la población de Argentina, es prioritario analizar a nivel intra-urbano la relación entre el suicidio y los fenómenos de fragmentación social y pobreza.

En los países latinoamericanos son escasos los estudios epidemiológicos dedicados al suicidio en áreas urbanas. Estos trabajos analizaron su evolución temporal en la CABA (Argentina) $)^{19}$, La Habana (Cuba) ${ }^{20}$, San Pablo (Brasil) ${ }^{21}$, Teresina (Brasil) ${ }^{22}$, Vitória (Brasil) ${ }^{23}$ y Medellín (Colombia $^{24}$, así como también su distribución geográfica en la CABA (Argentina) ${ }^{25}$ y la variación espacial de la densidad de suicidios en Porto Alegre (Brasil) ${ }^{26}$.

En general, los estudios ecológicos han mostrado mayores tasas de suicidio en áreas de menor nivel socioeconómico ${ }^{27}$. Dentro de estos estudios, 
son muy pocos los trabajos intra-urbanos espaciales, en general realizados en países desarrollados, y que también muestran este patrón ${ }^{28-30}$. En el caso de Latinoamérica, durante los últimos años se han publicado una serie de investigaciones dedicadas al análisis geográfico del suicidio, mayoritariamente realizadas en $\mathrm{Brasi}^{11-38}$. Sin embargo, los estudios ecológicos espaciales intra-urbanos son muy raros: solo dos investigaciones encontradas en bases de datos bibliográficas fueron llevadas a cabo en la ciudad de San Pablo, en Brasil ${ }^{39,40}$. Desde nuestro conocimiento, no existen estudios que analicen simultáneamente los efectos de la pobreza y la fragmentación social sobre la distribución geográfica del riesgo de suicidio a nivel urbano en Argentina.

Por lo tanto, el objetivo de este estudio es analizar la relación de la mortalidad por suicidio con el nivel socioeconómico y la fragmentación social en áreas pequeñas de la CABA durante el período 2011-2015. De acuerdo a los antecedentes encontrados, este estudio pone a prueba la siguiente hipótesis: se espera encontrar una mayor ocurrencia de suicidios en áreas con mayores índices de fragmentación social y menor nivel socioeconómico.

\section{Métodos}

El área de estudio comprendió la CABA (2.890.151 habitantes) y sus 351 fracciones censales delimitadas de acuerdo al Censo Nacional de Población, Hogares y Vivienda de 2010 (CNPHV 2010). Las fracciones censales son unidades territoriales definidas de acuerdo a la cantidad de viviendas. Estas unidades territoriales cuentan con un promedio de 5.000 viviendas.

Los estudios sobre la epidemiología del suicidio en Argentina utilizaron predominantemente datos de mortalidad provenientes del Ministerio de Salud de la Nación ${ }^{25,41-43}$. En el caso de la CABA, al considerar los casos de suicidio disponibles en la Dirección de Estadísticas e Información de Salud (DEIS) del Ministerio de Salud de la Nación, se detectó un marcado fenómeno de subregistro de casos de suicidio durante el período 2004-2014, con un promedio de 183 suicidios anuales entre 2004-2008 y entre 7 y 27 suicidios anuales durante el período 2011-2014. Esta disminución estuvo acompañada de un incremento de las defunciones por causas externas, cuya intencionalidad se desconoce. Por otro lado, el promedio anual de casos de suicidio reportados al Ministerio de Seguridad de la Nación durante
2011-2015 (150 casos) se asemeja al promedio anual de casos durante 2004-2008. Por lo tanto, la información disponible sobre mortalidad por suicidio de los últimos años no permitió realizar un estudio de su epidemiología del suicidio, por lo que se utilizaron los datos recabados por la Policía Federal Argentina disponibles en la Dirección Nacional de Gestión de la Información Criminal (DNGIC) del Ministerio de Seguridad de la Nación durante el período 2011-2015. En esos años fueron registrados 796 suicidios de residentes en CABA, de los cuales se lograron georreferenciar 742 casos (93\%). Los suicidios fueron georreferenciados mediante el complemento MMQGIS, disponible en el programa QGIS, y asignados a cada fracción censal.

El nivel de pobreza fue calculado con base al indicador elaborado por Carstairs \& Morris $^{44}$. Se calculó un índice modificado con las siguientes variables del CNPHV 2010: porcentaje de hombres desempleados en edad activa (14-64 años); porcentaje de personas en situación de hacinamiento (más de 3 personas por cuarto); porcentaje de jefes de hogar clasificados como 'obrero o empleado', 'trabajador por cuenta propia' o 'trabajador familiar' en la variable de categoría ocupacional; porcentaje de personas residiendo en viviendas de baja calidad (viviendas de categorías III y IV de acuerdo al CNPHV 2010). Las cuatro variables fueron transformadas en puntajes $z \mathrm{y}$ se realizaron sumas aritméticas de estos puntajes para formar un indicador de pobreza en cada fracción censal. Los puntajes $z$ fueron calculados como: $z=\chi-\mu$, donde $\chi$ es un valor de la variable, $\mu$ es el promedio de la variable, y $\sigma$ es el desvío estándar de la variable. Es así como los valores negativos indican niveles bajos de pobreza en una fracción censal, mientras que valores positivos indican niveles altos de pobreza.

El nivel de fragmentación social fue medido con variables del CNPHV 2010 tomando como base el índice desarrollado por Congdon ${ }^{28}$. Siguiendo la misma metodología que en el caso del indicador de pobreza, se calculó una versión modificada del índice de fragmentación social a través de la transformación en puntajes $z$ de las siguientes variables: porcentaje de adultos (mayores de 17 años) solteros, divorciados, separados legalmente o viudos; porcentaje de hogares conformados por una persona; porcentaje de hogares ocupados por no propietarios de vivienda; y porcentaje de población migrante (individuos mayores de 5 años de edad, quienes 5 años antes de la realización del censo residían en otra localidad). Se calcularon correlaciones entre estas va- 
riables y todas resultaron significativamente positivas, con los valores más altos de coeficientes de correlación entre el porcentaje de población migrante y el porcentaje de hogares conformados por una persona $(r=0,72)$, y entre ésta variable y porcentaje de hogares ocupados por no propietarios de vivienda $(r=0,64)$. La suma aritmética de los puntajes $z$ de cada variable dio como resultado un índice de fragmentación social para cada fracción censal. Es decir, valores negativos de este índice indicarían mayores niveles de integración social en un área determinada. En una fracción censal no hubo datos disponibles para las variables de migración y estado civil, por lo que se calculó un promedio de los valores porcentuales de las fracciones censales vecinas. Las variables relacionadas al tamaño de los hogares y al estado civil fueron seleccionadas como proxy del nivel de integración en la vida familiar y el matrimonio. Las variables referidas a la migración y al régimen de tenencia de las viviendas intentaron captar fenómenos de movilidad de la población, que al ser más intensos pueden promover menor integración social. Mientras la variable de migración capta movimientos de población más allá de la CABA, la inclusión de la variable de tipo de tenencia de la vivienda intenta captar movimientos intra-urbanos de población (áreas con alto porcentaje de viviendas ocupadas por inquilinos presentarían mayores niveles de movilidad poblacional). La elección de estas variables responde a su disponibilidad en el CNPHV 2010, por lo que otros indicadores relacionados con el nivel de integración social y que han tenido relación con la ocurrencia de suicidios, como los niveles de integración política y religiosa ${ }^{32,45,46}$, no pudieron ser incluidos en este estudio debido a su falta de disponibilidad al nivel de fracciones censales.

Se llevó a cabo una regresión múltiple espacial asumiendo una distribución de Poisson en los casos de suicidio, mientras que los índices de pobreza y fragmentación social fueron incorporados como variables independientes. Debido a que la variable dependiente consiste en los óbitos totales de suicidio (todas las edades y ambos sexos), se decidió incluir variables independientes relacionadas a la estructura demográfica de cada unidad espacial. Para ello se llevó a cabo un análisis de componentes principales con el fin de reducir las características relacionadas a la distribución por sexo y grupos de edad y así evitar la presencia de colinealidad en las variables independientes. Se incluyeron las siguientes variables: porcentaje de población entre 10 y 29 años, porcentaje de población entre 30 y 59 años, porcentaje de población mayor de 59 años e índice de masculinidad (porcentaje de varones sobre el porcentaje de mujeres). De dicho análisis se extrajeron los primeros dos componentes, ya que ambos explicaron el $77 \%$ de la varianza total (Tabla 1). El componente demográfico 1 estuvo caracterizado por áreas con alta proporción de población entre 10 y 59 años de edad y un índice bajo de masculinidad. En cambio, el componente demográfico 2 estuvo caracterizado por áreas con baja proporción de población mayor de 59 años, alta proporción de población de 10 a 29 años y un alto índice de masculinidad (Tabla 1). El análisis de componentes principales fue realizado con el programa Stata versión 13.1 (StataCorp, College Station, Texas, Estados Unidos).

Se calculó una razón de mortalidad, entre los casos de suicidio observados durante el período 2011-2015 y los casos esperados. Estos últimos fueron calculados considerando la población total residente multiplicada por cinco en cada fracción censal (debido a que son suicidios notificados durante cinco años), de acuerdo al censo de $2010^{47}$, y por la tasa de mortalidad por suicidio total de CABA.

Para incorporar la dependencia espacial entre las áreas, se empleó el modelo propuesto por Besag-York-Mollie (BYM) ${ }^{48}$. De esta manera, se incluye un parámetro que toma en cuenta los residuos espacialmente estructurados más un parámetro de residuos no estructurados. Los residuos espacialmente estructurados fueron calculados a través de una matriz de contigüidad espacial, en donde el criterio de vecindad fue determinado si un área compartía un límite con otra área. Se especificaron distribuciones a priori mínima-

Tabla 1. Coeficientes factoriales, valores propios y variabilidad explicada de los componentes principales utilizando variables demográficas del censo nacional de población. CABA, 2010.

\begin{tabular}{lcc}
\hline & $\begin{array}{c}\text { Componente } \\
\text { demográfico } \\
\mathbf{1}\end{array}$ & $\begin{array}{c}\text { Componente } \\
\text { demográfico } \\
\mathbf{2}\end{array}$ \\
\hline Población 10-29 años & 0,52 & 0,61 \\
Población 30-59 años & 0,68 & 0,13 \\
Población $\geq 60$ años & 0,42 & $-0,63$ \\
Índice de masculinidad & $-0,31$ & 0,45 \\
Valor propio & 1,97 & 1,11 \\
Variabilidad explicada & 50 & 28 \\
(\%) & & \\
\hline
\end{tabular}

Fuente: Censo Nacional de Población, Hogares y Viviendas 2010, Instituto Nacional de Estadística y Censos. 
mente informativas en el logaritmo de precisión del efecto no estructurado $\left(\tau_{v}\right) \sim \log$ Gamma $(1$, 0,0005), y el logaritmo de precisión del efecto estructurado $\left(\tau_{\mathrm{u}}\right) \sim \log \operatorname{Gamma}(1,0,0005)^{48}$. Se calcularon los riesgos relativos tomando en cuenta los dos efectos aleatorios. También se calcularon los riesgos relativos residuales, luego de tomar en cuenta las variables independientes incluidas en el modelo. Todas las variables independientes fueron introducidas en el modelo como variables cuantitativas continuas. Por último, se estimó la fracción de varianza espacial como:

$$
s_{u}^{2} /\left(s_{u}^{2}+\sigma_{v}^{2}\right) \text {, }
$$

donde $\mathrm{s}^{2}$ es la estimación empírica de la varianza marginal posterior para el efecto estructurado, y $\sigma_{v}^{2}$ es la varianza del componente marginal no estructurado ${ }^{48}$. Los análisis de regresión espacial se llevaron a cabo utilizando el paquete INLA en el programa $R$ (versión R.3.2.5), mientras que el mapeo de los índices de fragmentación social y pobreza, riesgos relativos y excesos de riesgo se realizó con el programa QGIS (versión 2.14.3).

La base de datos no contenía los nombres y apellidos de las víctimas ni elementos que pudieran identificarlas, y las muertes fueron agregadas a nivel de fracción censal, por lo que se cumple con los principios éticos para la investigación médica sobre sujetos humanos.

\section{Resultados}

$\mathrm{Al}$ analizar las tasas de suicidio en CABA por sexo y grupo etario durante el período 2011-2015, en general se observó una tasa creciente de suicidio a medida que aumentaba la edad (Tabla 2). Se registraron mayores casos y tasas de suicidio tanto en la población total como en mujeres y varones (Tabla 2). Las Figuras 1A-B muestran la distribución geográfica de los casos de suicidio y de su frecuencia relativa con respecto al total de sui- cidios notificados en CABA, respectivamente. $\mathrm{Al}$ observar la distribución geográfica de las tasas de mortalidad por suicidio, se pueden observar conjuntos de áreas contiguas de mayor mortalidad (Figura 1C). Se puede observar un patrón lineal de alta mortalidad a lo largo de los barrios Monserrat, Balvanera, Almagro y Caballito, y coincidente con la Avenida Rivadavia y la línea del ferrocarril Sarmiento de Estación Once (Figura 1). Otra zona de alta mortalidad se ubica desde los bosques de Palermo hacia Villa Crespo, en dirección a las avenidas Sarmiento/calle Thames hasta Warnes. Esta área también sigue la dirección del ferrocarril San Martin.

La Figura 1D muestra la distribución geográfica del riesgo relativo de muerte por suicidio a nivel de fracción censal, en comparación al riesgo total de la CABA, tomando en cuenta los efectos aleatorios estructurados y no estructurados. Se observa una concentración de valores altos en la mitad este de CABA. En cuanto a las variables independientes, el índice de fragmentación social muestra una concentración espacial de valores positivos en el centro este de la ciudad y valores negativos en las periferias del oeste, norte $y$ sur de CABA (Figura 2A). En general, el índice de pobreza muestra un gradiente de aumento en dirección norte a sur (Figura 2B). La Figura 1E muestra los riesgos relativos residuales luego de tomar en cuenta los componentes demográficos, el índice de fragmentación social y el índice de pobreza. En comparación a la Figura 1D, ya no se observa un patrón homogéneo de altos riesgos relativos en la mitad este de la CABA. En cambio, la nueva distribución geográfica parecería registrar una concentración de riesgos relativos altos en la zona suroeste de la ciudad (Figura 1E). Sin embargo, no se observaron probabilidades posteriores altas de encontrar riesgos relativos mayores a 1 , con valores de probabilidad que no superaron el 63\% (Figura 1F).

Tabla 2. Defunciones y tasas de suicidio por sexo y grupos de edad. CABA, 2011-2015.

\begin{tabular}{llrrrrrrr}
\hline & & \multicolumn{7}{c}{ Grupos de edad (años) } \\
\cline { 3 - 8 } & & $\mathbf{1 0 - 1 9}$ & $\mathbf{2 0 - 2 9}$ & $\mathbf{3 0 - 3 9}$ & $\mathbf{4 0 - 4 9}$ & $\mathbf{5 0 - 5 9}$ & $\mathbf{6 0 - 6 9}$ & $\mathbf{7 0 +}$ \\
\hline Defunciones & Ambos sexos & 26 & 121 & 131 & 87 & 107 & 95 & 166 \\
& Mujeres & 6 & 32 & 35 & 32 & 43 & 36 & 55 \\
& Varones & 20 & 89 & 96 & 55 & 64 & 59 & 111 \\
\multirow{2}{*}{ Tasas por 100000 } & Ambos sexos & 1,6 & 5,1 & 5,7 & 4,9 & 6,4 & 6,8 & 9,6 \\
habitantes & Mujeres & 0,8 & 2,6 & 2,9 & 3,4 & 4,7 & 4,4 & 4,9 \\
& Varones & 2,5 & 7,7 & 8,6 & 6,7 & 8,6 & 10,0 & 18,6 \\
& Po, & &
\end{tabular}

Fuente: Censo Nacional de Población, Hogares y Viviendas 2010, Instituto Nacional de Estadística y Censos; Dirección Nacional de Gestión de la Información Criminal. 

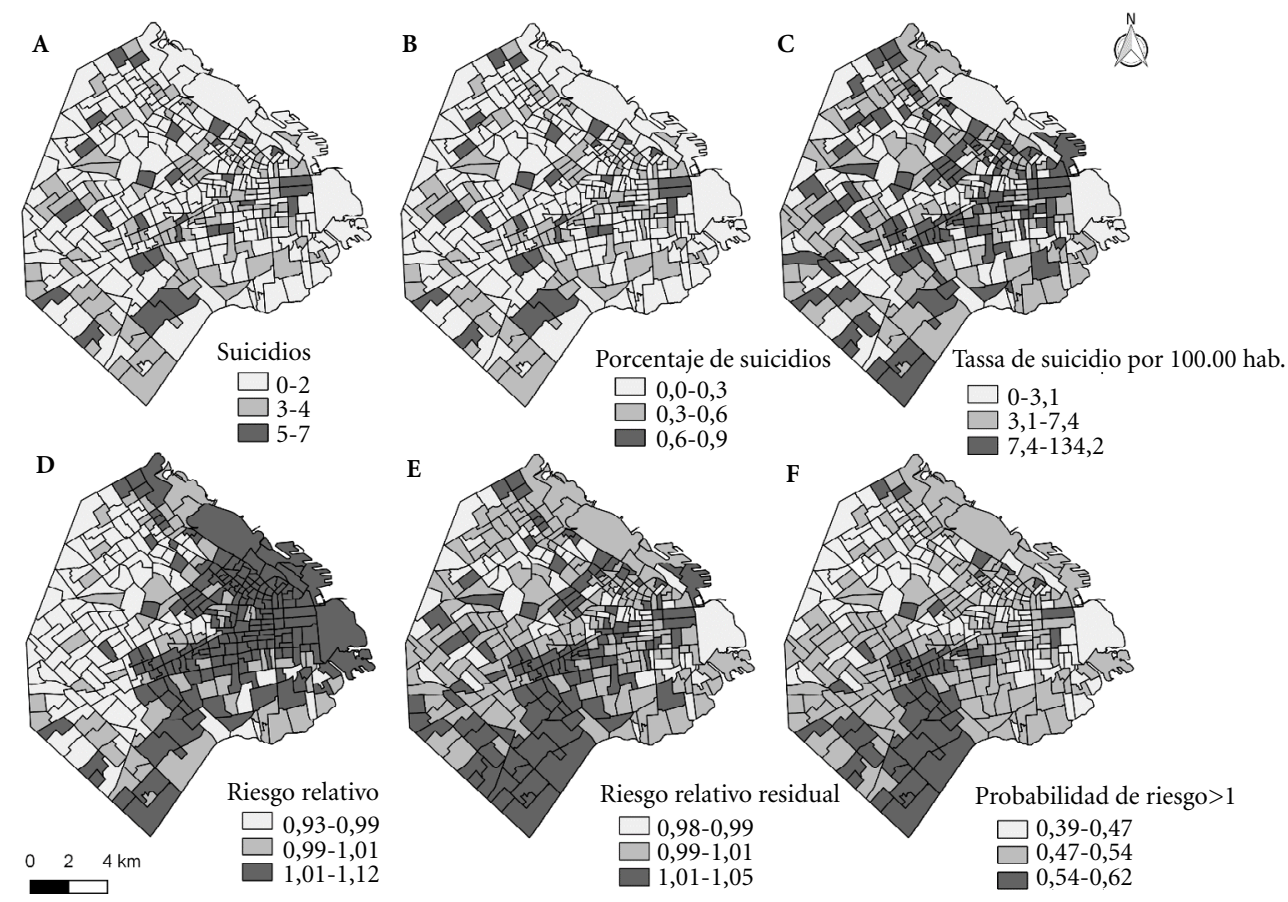

Figura 1. Distribución geográfica de los casos de suicidio (A), su porcentaje con respecto al total de suicidios en CABA (B), la tassa de suicidio (C), riesgo relativo de suicidio (D), riesgo relativo residual de suicidio (E) (luego del ajuste por las variables independientes del modelo), ambos comparados con el conjunto de CABA, y probabilidad posterior de riesgo $>1$ (F), 2011-2015.

Fuente: Censo Nacional de Población, Hogares y Viviendas 2010, Instituto Nacional de Estadística y Censos; Dirección Nacional de Gestión de la Información Criminal.
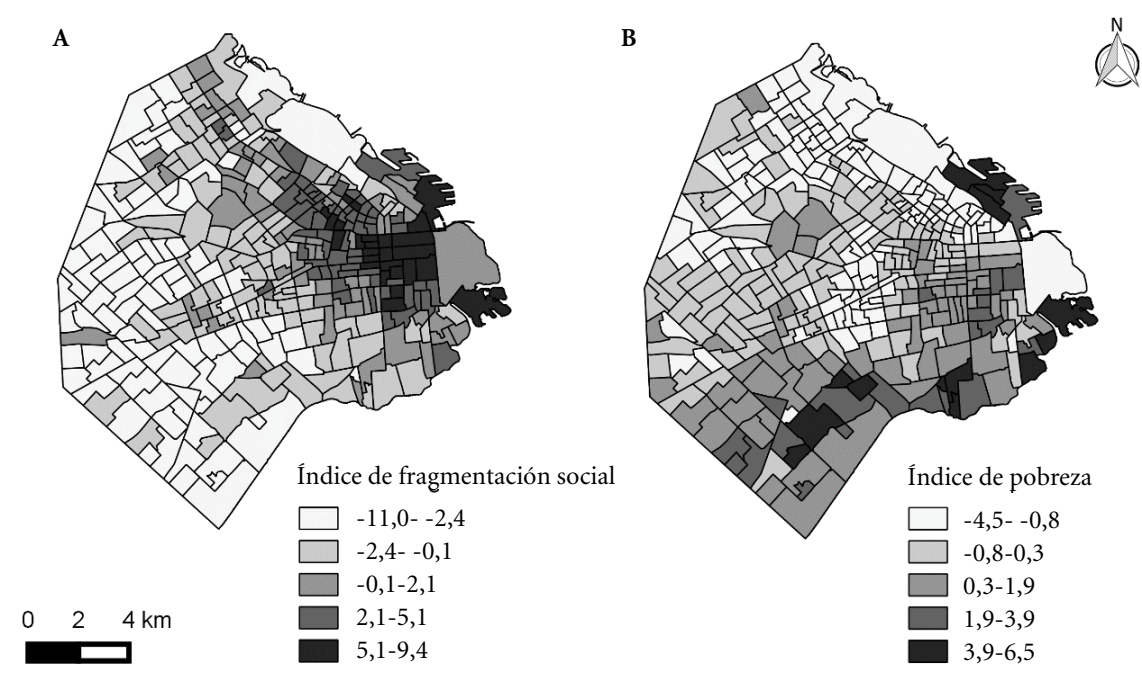

Figura 2. Distribución geográfica de los índices de fragmentación social (A) y pobreza (B). CABA, 2010.

Fuente: Censo Nacional de Población, Hogares y Viviendas 2010, Instituto Nacional de Estadística y Censos; Dirección Nacional de Gestión de la Información Criminal. 
La Tabla 3 muestra los resultados del modelo de regresión espacial. Controlando por los componentes demográficos, el riesgo de suicidio en relación al riesgo global de CABA estuvo asociado a niveles altos de fragmentación social y no se encontró asociación con el índice de pobreza (Tabla 3). Un aumento de 1 punto en el índice de fragmentación social estuvo asociado a un aumento del 5\% del riesgo relativo de suicidio. Por último, se calculó la fracción de varianza espacial, que pasó de 0,85 a 0,69 luego de incorporar las variables independientes al modelo. A pesar de haber disminuido, este último resultado sigue sugiriendo que una mayor parte de la varianza es explicada por el componente espacialmente estructurado del modelo.

\section{Discusión}

Las tasas de suicidio en CABA variaron geográficamente durante 2011-2015, concentrándose en determinadas áreas de la ciudad. Los resultados de este estudio también muestran que variables de área relacionadas a la fragmentación social se asociaron positivamente con la ocurrencia del suicidio a nivel intra-urbano, en una de las ciudades más densamente pobladas de Latinoamérica. Por otro lado, el indicador de pobreza no estuvo asociado con la ocurrencia de suicidio. Desde nuestro conocimiento, este es el primer estudio que analiza el impacto de ambos fenómenos sobre la distribución geográfica del suicidio en una ciudad argentina.

Tabla 3. Riesgos relativos (e intervalos de credibilidad del 95\%) y desvíos estándar del intercepto y las variables independientes.

\begin{tabular}{|c|c|c|}
\hline & Riesgo relativo $^{1}$ & $\begin{array}{c}\text { Desvío } \\
\text { Estándar }\end{array}$ \\
\hline Intercepto & $1,00(0,92-1,07)$ & 0,04 \\
\hline $\begin{array}{l}\text { Índice de } \\
\text { fragmentación social }\end{array}$ & $1,05(1,02-1,08)$ & 0,01 \\
\hline Índice de pobreza & $1,02(0,93-1,11)$ & 0,04 \\
\hline $\begin{array}{l}\text { Componente } \\
\text { demográfico } 1^{2}\end{array}$ & $1,02(0,94-1,12)$ & 0,05 \\
\hline $\begin{array}{l}\text { Componente } \\
\text { demográfico } 2^{3}\end{array}$ & $0,95(0,82-1,09)$ & 0,07 \\
\hline
\end{tabular}

${ }^{1}$ Ajuste multivariado. ${ }^{2}$ Componente caracterizado por áreas con alta proporción de población entre 10 y 59 años de edad y un índice bajo de masculinidad. ${ }^{3}$ Componente caracterizado por áreas con baja proporción de población mayor de 59 años, alta proporción de población de 10 a 29 años y un alto índice de masculinidad.
La distribución geográfica del suicidio en la ciudad de Buenos Aires muestra una concentración alta en áreas centrales del sector económico de servicios de la ciudad. Este patrón de distribución geográfica es similar al encontrado en otros estudios ecológicos que analizaron la variación intra-urbana del suicidio ${ }^{15,16,39}$. Las zonas centrales de mega-ciudades como Buenos Aires se caracterizan por un mayor uso del suelo comercial y variedad de actividades comerciales y un bajo uso residencial del suelo con respecto a las zonas periféricas urbanas. Esta diferenciación intra-urbana centro-periferia podría asociarse a altos niveles de fragmentación social en la zona central de la ciudad.

La relación positiva entre fragmentación social y suicidio apoya la hipótesis planteada por Durkheim: el suicidio varía inversamente con el grado de integración de los grupos sociales de los que el individuo forma parte ${ }^{3}$. Si espacializamos esta relación entre ambos fenómenos, podemos plantear la hipótesis de una distribución bimodal del riesgo de suicidio a lo largo de un gradiente de urbanización: alto riesgo de suicidio en áreas rurales (baja densidad poblacional y menor integración social) y en áreas con niveles altos de urbanización (alta densidad poblacional y menor integración social).

Los resultados del análisis de regresión espacial concuerdan con otro estudio latinoamericano en donde se encontró una relación positiva entre indicadores de fragmentación social (población migrante y soltera) y aquellos distritos en donde se localizó un conglomerado de alta mortalidad por suicidio ${ }^{40}$. Además, la relación positiva entre suicidio y fragmentación social fue reportada en estudios de áreas pequeñas llevados a cabo en países desarrollados ${ }^{28,30}$. Esta relación positiva también persiste tomando unidades espaciales mayores, como las unidades geográficas de segundo orden clasificadas por las oficinas de estadísticas de cada país ${ }^{6,45,46}$.

Si bien en nuestro estudio no se encontró una relación entre suicidio y pobreza, los resultados encontrados pueden interpretarse en relación a lo hallado en la ciudad brasileña de San Pablo, en donde se registró una relación positiva entre los ingresos por hogar y la presencia de conglomerados de alta mortalidad ${ }^{40}$. Ambos hallazgos contradicen lo hallado predominantemente en estudios ecológicos del suicidio utilizando unidades espaciales pequeñas en países desarrollados ${ }^{29,30}$. De acuerdo a Vijayakumar y colaboradores ${ }^{49}$, una posible explicación de este cambio en la relación entre suicidio y pobreza en países latinoameri- 
canos se encuentra en el rol del catolicismo. Sin embargo, la irrupción de otras religiones podría ser un factor que disminuye la tasa de suicidio en áreas de menor nivel socioeconómico. Estudios realizados en Brasil mostraron un menor riesgo de suicidio en áreas con mayor porcentaje de personas adherentes al pentecostalismo ${ }^{32,40}$. En el caso argentino, el crecimiento de pentecostalismo principalmente en la población de menor nivel socioeconómico ${ }^{50}$ podría actuar como un fenómeno que posibilite mayor integración social.

Una mayor proporción de la varianza explicada por el componente espacial del modelo de regresión apoya la hipótesis de concentración espacial de otros factores no tenidos en cuenta en el modelo de regresión. Las tasas de mortalidad en adultos mayores fueron las más altas, por lo que la inclusión de otros factores relacionados a condiciones socioeconómicas y de aislamiento social de estos grupos podría haber captado una mayor variabilidad de la distribución geográfica del suicidio en CABA. Este mayor riesgo de mortalidad de los adultos mayores, con respecto a grupos etarios más jóvenes en áreas urbanas, también ha sido reportado en ciudades de otros países ${ }^{20,51}$. Aunque las tasas de mortalidad en adultos mayores suelen ser mayores en áreas rurales con respecto a las áreas urbanas ${ }^{52,53}$, estudios futuros deberían analizar si estas brechas se observan en Argentina y cómo ha sido la evolución de éstas en el tiempo.

Otras investigaciones realizadas a nivel de país encontraron la influencia de variables medioambientales sobre la variación espacial de las tasas de suicidio. En Austria tanto la altitud como la presencia de litio en agua estuvieron asociados positiva y negativamente con las tasas de suicidio, respectivamente ${ }^{54}$. La persistencia de dependencia espacial, evidenciada a través del calculó de la fracción de varianza espacial, también permite plantear la hipótesis de fenómenos de imitación del suicidio en CABA. Esta hipótesis es más plausible teniendo en cuenta el uso de unidades espaciales pequeñas a nivel intra-urbano.

Este estudio presentó algunas limitaciones. En primer lugar, se utilizaron datos de suicidios provenientes del Ministerio de Seguridad, diferentes a los usualmente utilizados en estudios de suicidio en Argentina. Sin embargo, el patrón espacial encontrado en este estudio guarda similitud con la distribución geográfica de las tasas de mortalidad por suicidio, mediante el análisis de informes estadísticos de defunción, en CABA durante los años 1988, 1991, 2001 y 2002 ${ }^{25}$. De manera similar, se registró una concentración espacial de riesgos relativos altos de mortalidad en el centro-este de CABA. Segundo, se utilizaron datos censales de 2010 para el período de estudio 2011-2015, por lo que no se contó con datos precisos poblacionales anuales en cada fracción censal.

El análisis geográfico del suicidio mostró una concentración espacial del riesgo de suicidio en la zona este de CABA. Como se mencionó en el párrafo anterior, esta variabilidad geográfica también parece ser estable durante las últimas décadas. Un estudio realizado en Finlandia mostró una asociación entre la predominancia de servicios ambulatorios de salud mental (versus los servicios para pacientes hospitalizados) y tasas bajas de suicidio ${ }^{55}$. De acuerdo a un análisis realizado del presupuesto en salud mental de CABA durante 2016, el 85\% fue destinado a cuatro hospitales monovalentes dedicados específicamente a la atención de la salud mental ${ }^{56}$. Tres de estos hospitales se encuentran en dos áreas contiguas del sur de CABA. La desigual distribución geográfica del suicidio en CABA sugiere la necesidad de reorientar parte de estos recursos hacia las áreas de concentración espacial de alto riesgo de suicidio (principalmente la zona este de la ciudad) y focalizados en dispositivos intermedios de salud mental ${ }^{56}$.

\section{Conclusión}

Los resultados de este estudio ecológico apoyan la hipótesis de fragmentación social como un factor asociado al aumento del riesgo de suicidio a nivel intra-urbano en ciudades de países en desarrollo. El análisis geográfico del suicidio mostró una concentración espacial del suicidio en determinadas áreas de CABA. Como se mencionó en el párrafo anterior, esta variabilidad geográfica también parece ser estable durante las últimas tres décadas.

En conclusión, tanto el riesgo de suicidio como el índice de fragmentación social se concentraron en el espacio, mostrando en general un solapamiento entre ambas distribuciones geográficas. Luego de controlar por la distribución por sexo y edad de las áreas y un indicador de pobreza, los niveles altos de fragmentación social estuvieron asociados a altos riesgos de suicidio. 


\section{Colaboradores}

CM Leveau participó en la concepción y el diseño del estudio, análisis e interpretación de los datos, y en el proceso de escritura del artículo. C Guevel participó en el diseño del estudio y en la revisión crítica del contenido intelectual del artículo. M Alazraqui participó en la adquisición de los datos y en la revisión crítica del contenido intelectual del artículo. Los tres autores aprobaron la versión final del manuscrito.

\section{Referencias}

1. World Health Organization (WHO), editor. Preventing suicide: a global imperative. Geneva: WHO; 2014 $89 \mathrm{p}$.

2. Ministerio de Salud de la Nación. Descripción epidemiológica de la mortalidad por lesiones de causas externas en Argentina. Enfermedades no transmisibles y factores de riesgo. Boletín de vigilancia 6. Buenos Aires: Ministerio de Salud de la Nación; 2013.

3. Durkheim É. Suicide: a study in sociology. London; New York: Routledge; 2002.

4. Hawton K, Harriss L, Hodder K, Simkin S, Gunnell D. The influence of the economic and social environment on deliberate self-harm and suicide: an ecological and person-based study. Psychol Med 2001;31:827836.

5. Middleton N, Evans J, Gunnell D. Social fragmentation, severe mental illness and suicide. Soc Psychiatry Psychiatr Epidemiol 2004; 39:165-170.

6. Congdon P. The Spatial Pattern of Suicide in the US in Relation to Deprivation, Fragmentation and Rurality. Urban Stud 2011; 48:2101-2122.

7. Monk M. Epidemiology of suicide. Epidemiol Rev 1987; 9:51-69.

8. Laurell AC. Sobre la concepción biológica y social del proceso salud enfermedad. En: Lo biológico y lo social - su articulación en la formación de personal de salud. Washington D.C.: Organización Panamericana de la Salud; 1994.

9. Soja EW. The socio-spatial dialectic. Ann Assoc Am Geogr 1980;70:207-225.

10. Morley SA. The income distribution problem in Latin America and the Caribbean. Santiago, Chile: United Nations; 2001. 169 p. (Libros de la CEPAL).

11. Francisco Lucotti. Alemania o Haití: así es la desigualdad entre barrios ricos y pobres de Buenos Aires [Internet]. Sputnik Mundo 2018 [citado 2019 Apr 17]. Disponible en: https://mundo.sputniknews.com/sociedad/201812111084066865-desigualdad-argentina -barrios-ricos-pobres-buenos-aires/

12. Judd F, Cooper A-M, Fraser C, Davis J. Rural suicide-people or place effects? Aust N Z J Psychiatry 2006;40:208-216.

13. Schmid CF. Suicide in Minneapolis, Minnesota: 192832. Am J Sociol 1933; 39:30-48.

14. Schmid CF, Arsdol MDV. Completed and Attempted Suicides: A Comparative Analysis. Am Sociol Rev 1955; 20:273.
15. Buclass D, Duffy JC. The ecological pattern of suicide and parasuicide in Edinburgh. Soc Sci Med 1978;12:241-253.

16. Middleton N, Sterne JAC, Gunnell D. The geography of despair among 15-44-year-old men in England and Wales: putting suicide on the map. J Epidemiol Community Health 2006; 60:1040-1047.

17. Gunnell D, Wheeler B, Chang S-S, Thomas B, Sterne JAC, Dorling D. Changes in the geography of suicide in young men: England and Wales 1981-2005. J Epidemiol Community Health 2012; 66:536-543.

18. United Nations Population Division (UNPD). World urbanization prospects: the 2014 revision.New York, USA: UNPD; 2014.

19. Mazzeo V. Mortalidad por Suicidio en la Ciudad de Buenos Aires. Su magnitud y características. Transformaciones en el período 1980-1991. Texto Inéd Junio. 1993.

20. Laferté Trebejo A, Laferté Trebejo LA. Comportamiento del suicidio en Ciudad de La Habana intervención de enfermería en la atención primaria de salud. Rev Cuba Enferm 2000;16:78-87.

21. Nejar KA, Benseñor IM, Lotufo PA. Sunshine and suicide at the tropic of Capricorn, São Paulo, Brazil, 1996-2004. Rev Saude Publica 2007;41:1062-1064.

22. Parente ACM, Soares RB, Araújo ARF, Cavalcante IS, Monteiro CFS. Caracterização dos casos de suicídio em uma capital do Nordeste Brasileiro. Rev Bras Enferm 2007; 60:377-381.

23. Bastos MJRP, Pereira JA, Smarzaro DC, Costa EF, Bossanel RCL, Oliosa DMS, Pereira JGP, Feitosa HN Costa MF, Oliveira FJP, Fávero JL, Maciel ELN. Ecological analysis of accidents and lethal violence in Vitória, Southeastern Brazil. Rev Saude Publica 2009; 43:123-132.

24. Hernandez RDM. Características del suicidio al final del siglo XX en Medellín. CES Med 2009;14:53-59.

25. Spinelli H, Alazraqui M, Macías G, Zunino M, Nadalich JC. Muertes violentas en la Ciudad Autónoma de Buenos Aires: una mirada desde el sector salud. Buenos Aires: Organización Panamericana de la Salud; 2005.

26. Santos SM, Barcellos C, Carvalho MS, Flôres R. Detecção de aglomerados espaciais de óbitos por causas violentas em Porto Alegre, Rio Grande do Sul, Brasil, 1996. Cad Saude Publica 2001; 17:1141-1151. 
27. Rehkopf DH, Buka SL. The association between suicide and the socio-economic characteristics of geographical areas: a systematic review. Psychol Med 2005; 36:145.

28. Congdon P. Suicide and Parasuicide in London: A Small-area Study. Urban Stud 1996; 33:137-158.

29. Hsu C-Y, Chang S-S, Lee EST, Yip PSF. Geography of suicide in Hong Kong: Spatial patterning, and socioeconomic correlates and inequalities. Soc Sci Med 2015; 130:190-203.

30. Yoon T-H, Noh M, Han J, Jung-Choi K, Khang Y-H. Deprivation and suicide mortality across 424 neighborhoods in Seoul, South Korea: a Bayesian spatial analysis. Int J Public Health 2015; 60:969-976.

31. Híjar M, Rascón RA, Blanco J, López MV. Los suicidios en México. Características sexuales y geográficas (1979-1993). Salud Ment 1996; 19:14-21.

32. Bezerra Filho JG, Werneck GL, Almeida RLF de, Oliveira MIV de, Magalhães FB. Estudo ecológico sobre os possíveis determinantes socioeconômicos, demográficos e fisiográficos do suicídio no Estado do Rio de Janeiro, Brasil, 1998-2002. Cad Saude Publica 2012; 28:833-44.

33. Macente LB, Zandonade E. Spatial distribution of suicide incidence rates in municipalities in the state of Espírito Santo (Brazil), 2003-2007: spatial analysis to identify risk areas. Rev Bras Psiquiatr 2012; 34:261269.

34. Mota AA. Análise geocartográfica multiescalar do suicídio na América Latina e Caribe. Rev Espaço e Geogr 2015; 18:279-301.

35. Orellana JD, Balieiro AA, Fonseca FR, Basta PC, Souza MLP de. Spatial-temporal trends and risk of suicide in Central Brazil: an ecological study contrasting indigenous and non-indigenous populations. Rev Bras Psiquiatr 2016; 38:222-230.

36. Santos EGO, Barbosa IR. Conglomerados espaciais da mortalidade por suicídio no nordeste do Brasil e sua relação com indicadores socioeconômicos. Cad Saude Colet 2017; 25:371-378.

37. Dantas AP, Azevedo UN, Nunes AD, Amador AE, Marques MV, Barbosa IR. Analysis of suicide mortality in Brazil: spatial distribution and socioeconomic context. Rev Bras Psiquiatr 2017;40:12-18.

38. Santos AD, Guimarães LML, Carvalho YF, Viana LC, Alves GL, Lima ACR, Santos MB, Góes MAO, Araújo KCGM. Spatial analysis and temporal trends of suicide mortality in Sergipe, Brazil, 2000-2015. Trends Psychiatry Psychother [Internet]. 2018 [citado 2018 Oct 8]. Disponible en: http:// www.scielo.br/scielo.php?script $=$ sci_arttext\&pi$\mathrm{d}=$ S2237-60892018005004103\&lng=en\&tlng=en

39. Bando DH, Brunoni AR, Benseñor IM, Lotufo PA. Suicide rates and income in São Paulo and Brazil: a temporal and spatial epidemiologic analysis from 1996 to 2008. BMC Psychiatry 2012; 12:127.

40. Bando DH, Moreira RS, Pereira JC, Barrozo LV. Spatial clusters of suicide in the municipality of São Paulo 1996-2005: an ecological study. BMC Psychiatry 2012; $12: 124$.

41. Serfaty EM, Andrade JH, Foglia VL, Masaútis AE, Negri GM. Suicidio en adolescentes y jóvenes de 10-24 años. Argentina, 1991-2000. Sinopsis 2006; 22:16-21.

42. Sola M. Sociodemografía del suicidio en la población adolescente y joven en Argentina, 1999-2007. Rev Argent Salud Publica 2011; 2:18-23.
43. Bella M. Análisis de la mortalidad por suicidio en niños, adolescentes y jóvenes. Argentina, 2005-2007. Arch Argent Pediatr 2013; 111:16-21.

44. Carstairs V, Morris R. Deprivation: explaining differences in mortality between Scotland and England and Wales. BMJ 1989; 299:886-889.

45. Baller RD, Richardson KK. Social Integration, Imitation, and the Geographic Patterning of Suicide. Am Sociol Rev 2002; 67:873.

46. Balint L, Dome P, Daroczi G, Gonda X, Rihmer Z. Investigation of the marked and long-standing spatial inhomogeneity of the Hungarian suicide rate: A spatial regression approach. J Affect Disord 2014; 155:180-185.

47. Instituto Nacional de Estadística y Censos. WebINDEC: Población / Censos [Internet]. [citado 2018 Nov 11]. Disponible en: https://www.indec.gob.ar/nivel3_ default.asp?id_tema_1 $=28$ id_tema $\_2=41$

48. Blangiardo M, Cameletti M, Baio G, Rue H. Spatial and spatio-temporal models with R-INLA. Spat Spatio-Temporal Epidemiol 2013;4:33-49.

49. Vijayakumar L, Nagaraj K, Pirkis J, Whiteford H. Suicide in Developing Countries (1): Frequency, Distribution, and Association with Socioeconomic Indicators. Crisis 2005; 26:104-111.

50. Levita G. Estructura social y pluralización religiosa: un análisis del nivel socioeconómico de los pentecostales. Soc Religión 2010; 20:140-156.

51. Lee J, Lee W-Y, Noh M, Khang Y-H. Does a geographical context of deprivation affect differences in injury mortality? A multilevel analysis in South Korean adults residing in metropolitan cities. $J$ Epidemiol Community Health 2014; 68:457-465.

52. Watanabe N, Hasegawa K, Yoshinaga Y. Suicide in later life in Japan: urban and rural differences. Int Psychogeriatr 1995; 7:253-261.

53. Li M, Katikireddi SV. Urban-rural inequalities in suicide among elderly people in China: a systematic review and meta-analysis. Int J Equity Health 2019;18:2.

54. Helbich M, Blüml V, Leitner M, Kapusta ND. Does altitude moderate the impact of lithium on suicide? A spatial analysis of Austria. Geospatial Health 2013; 7:209-218.

55. Pirkola S, Sund R, Sailas E, Wahlbeck K. Community mental-health services and suicide rate in Finland: a nationwide small-area analysis. The Lancet 2009;373:147-153.

56. Asociación Civil por la Igualdad y la Justicia (ACIJ). Presupuesto para el área de salud mental: análisis del proyecto 2016. Buenos Aires: ACIJ; 2015.

Artículo presentado en 10/12/2018

Aprobado en 26/08/2019

Versión final presentada en 28/08/2019

Editores Jefes: Romeu Gomes, Antônio Augusto Moura da Silva 